\title{
Resolution of the Abraham-Minkowski Dilemma
}

\author{
Stephen M. Barnett \\ Department of Physics, SUPA, University of Strathclyde, Glasgow G4 ONG, United Kingdom
}

(Received 7 October 2009; published 17 February 2010)

\begin{abstract}
The dilemma of identifying the correct form for the momentum of light in a medium has run for a century and has been informed by many distinguished contributions, both theoretical and experimental. We show that both the Abraham and Minkowski forms of the momentum density are correct, with the former being the kinetic momentum and the latter the canonical momentum. This identification allows us to explain why the experiments supporting each of the rival momenta gave the results that they did. The inclusion of dispersion and absorption provides an interesting subtlety, but does not change our conclusion.
\end{abstract}

DOI: 10.1103/PhysRevLett.104.070401

PACS numbers: 03.50.De, 42.50.Nn, 42.50.Wk

In the early part of the last century Abraham and Minkowski proposed rival forms for the momentum density of light propagating through a material medium. The Abraham form is $\mathbf{g}_{\mathrm{Abr}}=\mathbf{E} \times \mathbf{H} / c^{2}$, while that due to Minkowski is $\mathbf{g}_{\text {Min }}=\mathbf{D} \times \mathbf{B}$, so that the associated total momenta are

$$
\mathbf{p}_{\mathrm{Abr}}=\int d V \frac{\mathbf{E} \times \mathbf{H}}{c^{2}} \quad \mathbf{p}_{\mathrm{Min}}=\int d V \mathbf{D} \times \mathbf{B} .
$$

A substantial body of work in the intervening hundred years has been devoted to attempting to determine which of these is correct [1-3]. At the simplest level, this difference amounts to assigning to a single photon the momentum $\mathbf{p}_{\text {Abr }}=\mathbf{p}_{0} / n$ or $\mathbf{p}_{\text {Min }}=\mathbf{p}_{0} n$, where $\mathbf{p}_{0}=\hbar \mathbf{k}_{0}$ is the photon momentum in free space and $n$ is the refractive index of the medium. Powerful arguments have been advanced in favor of both momenta. In particular the uniform motion of the center of mass energy, as expressed in Newton's first law of motion, supports the Abraham momentum $[2,4]$. The recoil of an absorbing or radiating atom in a medium [5,6] and the phenomenon of diffraction [7], however, argue with equal weight for the Minkowski momentum. Application of the Lorentz force law to the problem confirms the validity of these conclusions [8]. A simple presentation of these arguments is given in [9]. The situation has not really been clarified by experimental evidence, with observations reported in support of both the Abraham [10] and the Minkowski momentum [11]. Most recently, Campbell et al. [12] confirmed that the photon recoil of an atom in a dielectric is indeed of the Minkowski form, but the experiment of She et al. [13], although not uncontroversial [14,15], presented evidence in favor of the Abraham form. Amazingly, our resolution of the dilemma supports all of the above cited work.

We have no wish to add to the confusion on this issue and so begin with a brief presentation of two of the compelling arguments in favor of the Abraham and Minkowski momenta, one for each of them. We start by considering a thought experiment in which a single photon travels towards and then through a block of transparent medium [4]. The required uniform motion of the center of mass energy means that, as the photon is slowed by propagation through the medium, the block is necessarily displaced in the direction of propagation of the light. If the photon is moving in the $z$ direction then the displacement of the block must be $[16,17]$

$$
\Delta z=(n-1) L \frac{\hbar \omega}{M c^{2}},
$$

where $M$ and $L$ are the mass and thickness of the block and $\omega$ is the angular frequency of the photon. This displacement implies a velocity and hence a momentum for the block. Applying global momentum conservation then leads us to infer that the momentum of the photon while it is inside the medium is

$$
p_{\text {phot }}=\frac{\hbar \omega}{c n},
$$

in agreement with the Abraham form. We can turn this argument around and ask what the effect might be on the displacement of the block if we were to assign to the photon the Minkowski momentum. As the Minkowski momentum is greater than that of the photon in free space, the block would then necessarily move in the opposite direction to that of the photon. This, however, would be at odds with Newton's first law of motion, as generalized to apply to mass energy. It may reasonably be argued, of course, that it is for experiment to decide the issue, but a result supporting the Minkowski momentum in this case would have to contend with this conflict with the precept of the uniform motion of an isolated system.

For our second example, we consider an atom of mass $m$ with a transition at angular frequency $\omega_{0}$ traveling through a medium with velocity $v$ away from a light source [6]. The atom can absorb a photon from the source if its velocity is such that the Doppler shift brings the light into resonance: 


$$
\omega_{0} \approx \omega\left(1-\frac{n v}{c}\right) .
$$

If we apply the conservation of energy and momentum to the absorption process then we are led directly to conclude that the momentum of the photon inside the medium is

$$
p_{\text {phot }}=\frac{\hbar \omega n}{c},
$$

which is the Minkowski form. As with our first example, it may be argued that it is for experiment to decide the issue. A result supporting the Abraham momentum in this case, however, would have to account for the mismatch between the change in the atomic momentum and that of the absorbed photon.

These arguments (and others we could have discussed) are both simple and compelling and it is hard to see how either could be incorrect and, indeed, neither is. We note that in dispersive media the phase index $\left(n_{p}=c k / \omega\right)$ and the group index $\left[n_{g}=c(d \omega / d k)^{-1}\right]$ will be different. It is clear from the above analyses that the Abraham momentum in this case will have magnitude $\hbar \omega / c n_{g}$ and that the Minkowski momentum should have the magnitude $\hbar \omega n_{p} / c$

It has been proposed that the origin of the distinction between the Abraham and Minkowski momenta is connected to the distinction in electromagnetism between the kinetic and canonical momenta [18-20]. It will be recalled that the kinetic momentum of a body is simply the product of its mass and velocity. In quantum theory, the canonical momentum of a body is simply Planck's constant divided by its de Broglie wavelength. It is the canonical momentum, of course, that appears in the canonical commutation relation $[\hat{x}, \hat{p}]=i \hbar$. The canonical momentum depends on the gauge chosen, but in the dipole form these two momenta, for a point dipole, are related by $[20,21]$

$$
\mathbf{p}_{\text {kin }}=\mathbf{p}_{\mathrm{can}}+\mathbf{d} \times \mathbf{B}-\frac{\mathbf{m} \times \mathbf{E}}{c^{2}},
$$

where $\mathbf{d}$ and $\mathbf{m}$ are the electric and magnetic moments, and $\mathbf{E}$ and $\mathbf{B}$ are the electric field and the magnetic induction at the position of the dipole. For a collection of such dipoles forming a medium we can add these momenta together to give the total momentum. The electric and magnetic fields include contributions from the surrounding dipoles but these cancel each other by virtue of Newton's third law of motion [22]. We are left with the unique total momentum for the medium and the field

$$
\mathbf{p}_{\mathrm{kin}}^{\text {med }}+\mathbf{p}_{\mathrm{Abr}}=\mathbf{p}_{\mathrm{can}}^{\text {med }}+\mathbf{p}_{\mathrm{Min}} .
$$

We can therefore unambiguously identify the Abraham and Minkowski momenta, respectively, with the kinetic and canonical momenta of the light. The total momentum (whether kinetic or canonical) is a single conserved quantity.
There are two remaining problems in the AbrahamMinkowski dilemma: why is it that the experiments supporting one or other of these momenta give the results that they do and how can we correctly identify the momenta in a dispersive medium [19]? Explaining these, we suggest, completes the resolution of the dilemma.

The kinetic momentum of a material body is simply the product of its mass and velocity. It is this quantity that appears in the above argument, based on uniform motion, in support of the Abraham momentum. We identify the momentum of the light as $\mathbf{p}_{0} / n$, or more precisely $\mathbf{p}_{0} / n_{g}$, for each photon by applying the conservation of (kinetic) momentum. It is for this reason that the kinetic momentum of the light, or the Abraham momentum, appears. It is straightforward to confirm, by direct calculation, that the single-photon expectation value of $\mathbf{p}_{\mathrm{Abr}}$, as defined in Eq. (1), is indeed $\mathbf{p}_{0} / n_{g}$ as it should be [19].

The nature of the canonical momentum is more subtle than its kinetic counterpart. It is intimately related to the idea of translations, indeed it is the infinitesimal generator of such translations within, or relative to, the host medium. We can investigate this idea using either a classical or a quantum theory but for definiteness, and appropriateness to the discussion of a single photon, choose the quantum description. In the Coulomb gauge the vector potential operator and the displacement operator satisfy the equaltime canonical commutation relation

$$
\left[\hat{A}_{j}(\mathbf{r}), \hat{D}_{k}\left(\mathbf{r}^{\prime}\right)\right]=-i \hbar \delta_{j k}^{\perp}\left(\mathbf{r}-\mathbf{r}^{\prime}\right),
$$

where $\delta_{j k}^{\perp}\left(\mathbf{r}-\mathbf{r}^{\prime}\right)$ is the transverse delta function [23]. We note that this commutation relation holds for dielectric media even if we include both dispersion and losses [24]. It is straightforward to use this to show that it is the Minkowski momentum that generates a translation of the vector potential:

$$
\exp \left(-i \frac{\mathbf{a} \cdot \hat{\mathbf{p}}_{\text {Min }}}{\hbar}\right) \hat{\mathbf{A}}(\mathbf{r}) \exp \left(i \frac{\mathbf{a} \cdot \hat{\mathbf{p}}_{\text {Min }}}{\hbar}\right)=\hat{\mathbf{A}}(\mathbf{r}+\mathbf{a}),
$$

where $\mathbf{a}$ is a constant vector. It follows that the Minkowski momentum is the canonical momentum. For a body immersed in a medium it is precisely this translation, relative to the medium that is important. It is for this reason that the Minkowski momentum appears in experiments that measure the displacement of a body embedded in a host $[11,12]$. The canonical momentum is also intimately related to the wavelength of the light in that a translation induces a phase shift proportional to the ratio of the position shift and the wavelength. This intimate connection is the reason that it is the canonical, or Minkowski, momentum that appears in the analysis of diffraction [7].

There is a remaining problem, which is that the singlephoton expectation value of $\hat{\mathbf{p}}_{\text {Min }}$ is not $n_{p}\left\langle\hat{\mathbf{p}}_{0}\right\rangle$, but rather $n_{p}^{2}\left\langle\hat{\mathbf{p}}_{0}\right\rangle / n_{g}$ [19]. We can see this directly by noting that the 
single-photon expectation value of $\hat{\mathbf{p}}_{\mathrm{Abr}}$ is $\left\langle\hat{\mathbf{p}}_{0}\right\rangle / n_{g}$ and that $\left\langle\hat{\mathbf{p}}_{\text {Min }}\right\rangle=\varepsilon \mu\left\langle\hat{\mathbf{p}}_{\text {Abr }}\right\rangle=n_{p}^{2}\left\langle\hat{\mathbf{p}}_{\text {Abr }}\right\rangle$. This has led to the suggestion that there is a difference between the canonical momentum, which takes the value $n_{p}\left\langle\hat{\mathbf{p}}_{0}\right\rangle$, and the Minkowski momentum, which seems to take the value $n_{p}^{2}\left\langle\hat{\mathbf{p}}_{0}\right\rangle / n_{g}$ [5,19]. In fact this is not the case and the Minkowski momentum is indeed $\hat{\mathbf{p}}_{0} n_{p}$. The explanation for this lies in the quite distinct roles of the kinetic and canonical momenta. The kinetic or Abraham momentum is the kinetic momentum carried by the photon and it is meaningful, therefore, to calculate it by taking the single-photon expectation value of $\hat{\mathbf{p}}_{\mathrm{Abr}}$. The canonical momentum, however, is associated with spatial translations and its defining property, therefore, is the translation in Eq. (9) or, equivalently, the commutation relation [25]

$$
\left[\hat{p}_{\text {Min }}^{j}, \hat{A}^{k}(\mathbf{r})\right]=i \hbar \frac{\partial}{\partial x^{j}} \hat{A}^{k}(\mathbf{r}) .
$$

For a field excitation at angular frequency $\omega$, this derivative results in multiplying the vector potential by $\hbar k^{j}=$ $n_{p} p_{0}^{j}$, which is the canonical or Minkowski momentum.

We can and should ask why the single-photon expectation value of $\hat{\mathbf{p}}_{\text {Min }}$ is not the canonical momentum. The answer to this question lies in the fact that electromagnetic quanta in the medium are polaritons (or similar species), that is excitations of the coupled optical and polarization (or magnetization) fields [26]. Each polariton mode contributes to the commutation relation, Eq. (8), its own value of $n_{p} / n_{g}$. The total for each value of $k$, however, is [24,27]

$$
\sum_{i} \frac{n_{p}\left(\omega_{i}\right)}{n_{g}\left(\omega_{i}\right)}=\sum_{i} \frac{k}{\omega_{i}} \frac{d \omega_{i}}{d k}=1,
$$

where $\omega_{i}$ are the solutions of the dispersion relation $\omega(k)=c k / n_{p}(\omega)$. An elementary example with just two polariton branches is depicted in Fig. 1. The commutation relations (10) and then the translation formula, Eq. (9),

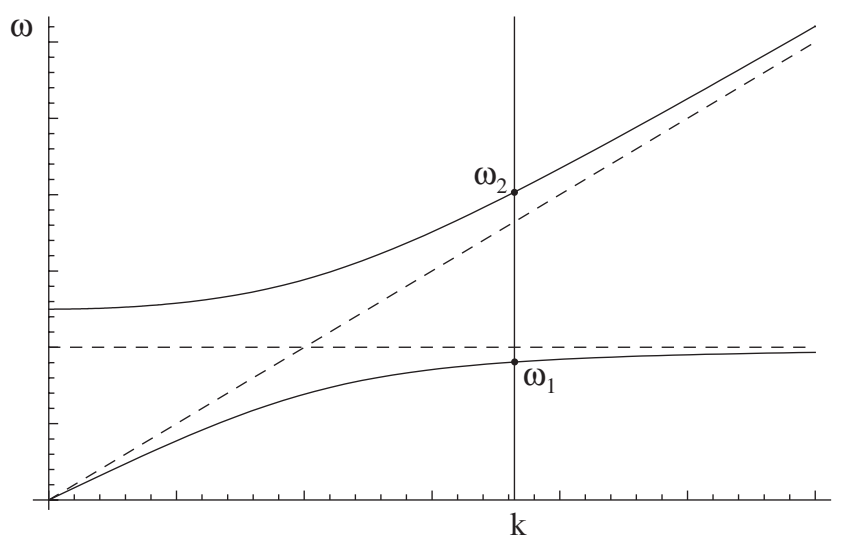

FIG. 1. A simple two-branch polariton dispersion curve. The two values of $\omega$ for each value of $k$ are constrained to satisfy the sum rule in Eq. (11) [24,27]. are consequences of the canonical commutation relation, Eq. (8), and therefore, of the sum rule in Eq. (11). Had we included only one of the polariton branches in our vector potential and displacement fields, then the commutator in Eq. (10) would be replaced by one in which each frequency component of $\hat{\mathbf{A}}$ would be multiplied by $n_{p} / n_{g}$ at that frequency. This, when combined with the derivative, would give an effective momentum with the value $n_{p}^{2} \hat{\mathbf{p}}_{0} / n_{g}$. The identification of this value with the Minkowski momentum is a result of making a narrow bandwidth approximation and thereby omitting all but one of the polariton branches. We might also ask whether by working with a suitably narrow bandwidth we might encounter a regime in which the canonical momentum is $n_{p}^{2} \hat{\mathbf{p}}_{0} / n_{g}$. Our analysis suggests that this is not the case. The translation of the fields, as embodied in Eq. (9), is a property of the commutation relations and is not dependent on any specific field state. We must conclude that the presence of all of the polariton modes, even if not excited, plays its part in the translation. The narrow bandwidth approximation is often useful, but retaining the modes corresponding to all frequencies is necessary if we are to retain the correct commutation properties.

Our resolution of the Abraham-Minkowski dilemma requires us to recognize that there are two distinct electromagnetic momenta, the kinetic momentum and the canonical momentum. The total momentum of matter plus light is unique, but the division of this into optical and material parts may be performed so as to separate kinetic or canonical parts. The Abraham and Minkowski momenta are, respectively, the kinetic and canonical optical momenta.

We conclude by noting that a number of further momenta have been proposed, with the aim of resolving the Abraham-Minkowski dilemma [2]. By demonstrating the need for two "correct" momenta and associating these, unambiguously, with the Abraham and Minkowski forms, we may hope that we have also removed the need for further rival forms.

I am grateful to many friends and colleagues from whom I have learnt so much about the Abraham-Minkowski dilemma, but especially to Rodney Loudon who first introduced me to this puzzle, and whose stimulating questions have guided this invesitgation. This work was supported by the U.K. Engineering and Physical Sciences Research Council, the Royal Society, and the Wolfson Foundation.

[1] U. Leonhardt, Nature (London) 444, 823 (2006).

[2] I. Brevik, Phys. Rep. 52, 133 (1979).

[3] R. N. C. Pfeifer, T. A. Nieminen, N. R. Heckenberg, and H. Rubinsztein-Dunlop, Rev. Mod. Phys. 79, 1197 (2007).

[4] N. L. Balazs, Phys. Rev. 91, 408 (1953).

[5] P. W. Milonni and R. W. Boyd, Laser Phys. 15, 1 (2005). 
[6] D. H. Bradshaw, Z. Shi, R. W. Boyd, and P. W. Milonni, Opt. Commun. 283, 650 (2010).

[7] M. J. Padgett, Opt. Express 16, 20864 (2008); I. Brevik, Eur. J. Phys. 2, 37 (1981).

[8] R. Loudon, S. M. Barnett, and C. Baxter, Phys. Rev. A 71, 063802 (2005).

[9] S. M. Barnett and R. Loudon, Phil. Trans. R. Soc. A 368, 927 (2010).

[10] G. B. Walker, D. G. Lahoz, and G. Walker, Can. J. Phys. 53, 2577 (1975).

[11] R. V. Jones and J. C. S. Richards, Proc. R. Soc. A 221, 480 (1954); A. Ashkin and J. M. Dziedzic, Phys. Rev. Lett. 30, 139 (1973); R. V. Jones and B. Leslie, Proc. R. Soc. A 360, 347 (1978); A. F. Gibson, M. F. Kimmitt, A. O. Koohian, D. E. Evans, and F. D. Levy, Proc. R. Soc. A 370, 303 (1980); M. Kristensen and J.P. Woerdman, Phys. Rev. Lett. 72, 2171 (1994).

[12] G. K. Campbell, A. E. Leanhardt, J. Mun, M. Boyd, E. W. Streed, W. Ketterle, and D. E. Pritchard, Phys. Rev. Lett. 94, 170403 (2005).

[13] W. She, J. Yu, and R. Feng, Phys. Rev. Lett. 101, 243601 (2008).

[14] M. Mansuripur, Phys. Rev. Lett. 103, 019301 (2009).

[15] I. Brevik, Phys. Rev. Lett. 103, 219301 (2009).

[16] O. R. Frisch, Contemp. Phys. 7, 45 (1965).

[17] R. Loudon, Fortschr. Phys. 52, 1134 (2004).

[18] C. Baxter, M. Babiker, and R. Loudon, Phys. Rev. A 47, 1278 (1993); U. Leonhardt, Phys. Rev. A 73, 032108 (2006).
[19] J. C. Garrison and R. Y. Chiao, Phys. Rev. A 70, 053826 (2004).

[20] E. A. Hinds and S. M. Barnett, Phys. Rev. Lett. 102, 050403 (2009).

[21] We note that this difference between the canonical and kinetic momenta has also been ascribed to a stress acting on the material medium itself, E. G. Cullwick, Proc. IEE 113, 369 (1966).

[22] S. M. Barnett and R. Loudon, J. Phys. B 39, S671 (2006).

[23] E. A. Power, Introductory Quantum Electrodynamics (Longmans, London, 1964).

[24] B. Huttner, J. J. Baumberg, and S. M. Barnett, Europhys. Lett. 16, 177 (1991); B. Huttner and S. M. Barnett, Phys. Rev. A 46, 4306 (1992).

[25] For completeness, we note that the analogous commutation relation for the Abraham momentum is

$$
\begin{aligned}
{\left[\hat{p}_{\mathrm{Abr}}^{j}, \hat{A}^{k}(\mathbf{r})\right]=} & i \hbar \frac{\partial}{\partial x^{j}} \hat{A}^{k}(\mathbf{r})-i \hbar \mu_{0} \varepsilon_{j l m} \\
& \times \int d V^{\prime} \delta_{l k}^{\perp}\left(\mathbf{r}-\mathbf{r}^{\prime}\right) \hat{M}^{m}\left(\mathbf{r}^{\prime}\right),
\end{aligned}
$$

which is the same as that given in Eq. (10) only if the magnetization $\hat{\mathbf{M}}$ is omitted.

[26] C. Kittel, Quantum Theory of Solids (Wiley, New York, 1987).

[27] O. Al-Dossary, M. Babiker, and N. Enfati, Phys. Rev. A 54, 2419 (1996). 